\title{
Perceived facilitators and barriers to chronic disease management in primary care networks: a qualitative study
}

Chuan De Foo ( $\nabla$ ephfchu@nus.edu.sg )

National University of Singapore https://orcid.org/0000-0001-7254-4881

Shilpa Surendran

National University Singapore Saw Swee Hock School of Public Health

Chen Hee Tam

National University Singapore Saw Swee Hock School of Public Health

Elaine Qiao Ying Ho

National University Singapore Saw Swee Hock School of Public Health

David Bruce Matchar

Duke-NUS Medical School

Josip Car

Lee Kong Chian School of Medicine

Gerald Choon Huat Koh

National University Singapore Saw Swee Hock School of Public Health

Research article

Keywords: General practitioner, primary care network, qualitative study

Posted Date: June 4th, 2020

DOI: https://doi.org/10.21203/rs.3.rs-28942/v1

License: (c) (i) This work is licensed under a Creative Commons Attribution 4.0 International License.

Read Full License 


\section{Abstract \\ Background}

The increasing chronic disease burden in developed countries has placed tremendous strain on tertiary healthcare infrastructure and resources. Therefore, there is an urgent need to shift chronic disease management from tertiary to primary care providers to mitigate the increase in demand for chronic care at hospitals. The organization of private general practitioners (GPs) into Primary Care Networks (PCNs) is a pragmatic move by Singapore, a developed and multi-ethnic urban city, to provide private GPs with team-based care capabilities and a platform to track care indicators for better management of chronic patients. As the PCN initiative is still in its embryonic stages, there is a void in research regarding its ability to empower private GPs to manage chronic patients effectively. This qualitative study aims to explore the facilitators and barriers for the management of chronic patients by private GPs in the PCN.

\section{Method:}

We conducted 30 semi-structured in-depth interviews with GPs enrolled in a PCN. Qualitative analysis of audio transcripts was performed to extract themes which highlighted the facilitators and barriers faced by PCN in the early stages of its development.

\section{Results}

Our results suggest that PCNs facilitated private GPs to more effectively manage chronic patients through 1) provision of ancillary services such as diabetic foot screening, diabetic retinal photography and nurse counselling to permit a "one-stop-shop", 2) systematic monitoring of process and clinical outcome indicators through a chronic disease registry (CDR) to promote accountability for patients' health outcomes and 3) funding streams for PCNs to hire additional manpower to oversee operations and to reimburse GPs for extended consultations. Barriers include high administrative load in maintaining the CDR due to the lack of a smart electronic clinic management system and financial gradient faced by patients seeking services from private GPs which incur higher out-of-pocket expenses than public primary healthcare institutions.

\section{Conclusion}

PCNs demonstrate great promise in empowering and motivating private GPs to manage chronic patients. However, barriers will need to be addressed to ensure the quality and comprehensiveness of PCNs in managing more chronic patients in the face of an ageing population.

\section{Introduction}


As the global population ages at an alarming pace, the number of chronic patients is set to rise in tandem. Both developing and developed countries such as the United States and United Kingdom are thus facing an unprecedented strain on their tertiary healthcare infrastructure resulting from the overutilization of healthcare services for the delivery of chronic care [1-5]. The unparalleled surge in demand for healthcare culminates into higher bed occupancy rates and emergency department presentations, which impose substantial expenditures on the healthcare system [4, 6, 7]. A systematic review by Reynolds et al. (2018) illustrated that chronic disease interventions through primary care interfaces such as self-management support and closer monitoring could potentially prevent $40 \%$ of hospitalizations in patients with multiple chronic conditions [8,9]. Shifting chronic disease management to the primary care sector is certainly no panacea. Nevertheless, this move will permit some level of cost containment, improved continuity of care and overall better health outcomes [10-13].

Care management is delineated by a set of interventions encompassing but not limited to holistic evaluation of patients' medical and nonmedical requirements, tracking of personalized, evidence-based treatment plans designed around joint disease management measures through a care team $[11,14,15]$. Based on concepts for the re-organization of chronic care, care management interventions have been developed to focus on patients with multiple comorbidities. The organization of primary care providers into networks potentially provides better health gains than individual practices and strengthens coordination with hospital and community partners. Migrating chronic disease management to primary care contexts have necessitated a more team-based approach whereby a multidisciplinary group of professionals operates with the primary care doctor to accomplish shared goals within and across settings to achieve higher quality care $[16,17]$.

The Primary Care Network (PCN) is a model of care that enshrines the delivery of team-based primary health services, through a team of physicians, nurse counsellors, care coordinators, and administrative assistants $[18,19]$. Such networks have been established in Canada, New Zealand and Germany since the early 2000s, and have led to improved patient access to primary care and quality of care [20-22]. In Germany, PCNs have displayed positive results in the management of chronic diseases such as diabetes by serving as a vehicle of care that focuses on improving access to care and chronic disease selfmanagement practices through the use of multidisciplinary teams $[22,23]$. PCNs implemented in Alberta had demonstrated their capacities in reducing presentations to emergency departments and hospital days for non-elective acute care, further emphasizing the significance of team-based care at the primary care interface [24, 25]. Two studies by Johnson et al. (2016) also demonstrated PCN's team-based approach to care for diabetic and depressed patients as a cost-effective management strategy [26, 27]. Furthermore, PCNs can facilitate sharing of resources, allowing for greater bargaining power when tendering for services, sharing expertise between parties and reducing the organizational workload of practices [28].

With the upsurge in chronic load, Singapore's healthcare system, which was historically constructed to cater for acute episodic conditions, is in need of a transformative change. As a result, the policy for PCN was promulgated as a primary care model by the Ministry of Health $(\mathrm{MOH})$ in 2018 , with hopes that the 
formalization of primary care ecosystems would serve as a conduit to evolve Singapore's primary care landscape to better manage patients with chronic conditions [29].

\section{Context of our study}

Singapore's healthcare system is accessible through an extensive network of hospital, step-down and primary care providers. The primary care sector is split between private and public run entities. Public healthcare institutions called polyclinics are government-funded, and patients enjoy substantial subsidies for their consultations, medications and diagnostic investigations. Additionally, patients are free to choose where to receive their care, either in the government-subsidized public sector or private sector for all levels of care. The healthcare financing model is primarily based on fee-for-service. Only in recent years, has the government provided subvention to the primary care sector through the introduction of the Community Health Assistance Scheme (CHAS). CHAS is a form of portable medical subsidies that enable Singaporean patients to enjoy a certain quantum provided by the government to offset medical expenses when seeking treatment at private primary care facilities. Singapore's $\mathrm{MOH}$ and its statutory boards such as the Agency of Integrated Care (AIC), oversee both public and private primary care providers and establish regulations and evidence-based standards that ensure healthcare's quality and affordability [30, 31].

At the primary care interface, private general practitioners (GPs), play a dominant role, with more than 1,700 private clinics offering primary care services [32]. Public primary care is provided by 20 polyclinics distributed throughout the island. Polyclinics are multi-doctor (usually more than 10) clinics that provide a comprehensive range of services for the family, functioning as a one-stop centre for acute and chronic conditions [33].

In terms of primary care utilization, private GPs provide $80 \%$ of all attendances out of which only $20 \%$ turn to them for the management of chronic conditions [34]. Coupled with the projected rise in chronic numbers, PCNs were commissioned to mobilize more private primary care sector resources. This was envisaged through the voluntary enrollment of GP practices into a PCN of their choice. As of February 2020, a total of 508 private GP practices have been enrolled [19]. Furthermore, within each PCN, two PCN leads who are doctors themselves, will be internally selected from their PCN to lead their network. Currently, a total of ten PCNs of varying sizes are under the purview of AIC, each a cluster of hitherto independent GP practices working more closely together through sharing of manpower and ancillary services to offer extended care for patients.

To our knowledge, there are only two quantitative studies conducted to evaluate the effectiveness of PCN in Singapore, both exclusively for diabetes management [35, 36]. However, there has been no qualitative study that investigates how this model of care facilitates or challenges the management of chronic diseases from the providers' perspective. Therefore, this study aims to understand the experiences of private GPs enrolled in PCNs and explore the facilitators and barriers of PCN in helping private GPs manage chronic disease patients. 


\section{Method}

\section{Study Design}

Our study employed a qualitative research design [37] using data collected from semi-structured in-depth interviews conducted with participants who met the inclusion criteria of being a private GP enrolled in a PCN. A qualitative research method was appropriate as the study intended to explore experiences of the private GPs on the research topic [38].

\section{Participants}

Purposive and snowball sampling strategies were employed to recruit eligible participants. Most participants were contacted via email or telephone, as most contact details were available on publicly accessible websites. Snowball sampling was useful in reaching out to eligible participants whose contact details were not made available on public domains. Only participants who had completed the interviews were asked to refer us to other eligible participants for snowball sampling.

A total of 30 eligible participants took part in the study while seven had refused participation with the common reason of having insufficient time to be interviewed. There was no form of reimbursement for their time. Nonetheless, if eligible participants did not respond to the initial recruitment email, another follow-up email or call would be made. They were not contacted after two attempts at recruitment. The characteristics of the participants are stated in the results section below.

\section{Research team}

The interviewers (CD, male and SS, female) had no relationship with the participants and the team that analyzed the audio transcripts had a profound knowledge of the Singapore healthcare system. Both interviewers were research associates trained in qualitative research and had obtained their Master of Public Health (MPH). However, two of the study team members were acquainted with some of the participants due to prior research studies conducted with them. These two team members were not involved in the interviews nor the analysis of the data.

\section{Data collection}

The semi-structured in-depth interviews were conducted at a place of the participants' convenience. The interviews took place between January 2019 and January 2020 and the duration of the interviews ranged from 40 to 90 minutes.

The topic guide (Additional file 1) was guided by our research question as we were looking at the enabling characteristics of PCNs which helped private GPs better manage patients with chronic conditions. The topic guide was pilot tested before implementation. As the interviews were semistructured, there were no restrictions on the conversation flow but the interviewers directed and adapted the conversation to elicit responses that could answer the research question. Field notes were collected during the interview. 
After the interviews, the audio recordings and subsequently, audio transcripts were de-identified to ensure that the data could not be traced to the participants. Member checking, whereby interview transcripts were sent back to participants to be reviewed, was not performed. The consolidated criteria for reporting qualitative research (COREQ) criteria (Additional file 2) was applied to this manuscript [39].

\section{Data analysis}

All audio recordings were transcribed verbatim, and the transcripts were analyzed by CD and SS using a phenomenological approach [40] with QSR NVivo software (version 12) following a thematic process outlined by Braun and Clark [41]. As such, the "weightage" of a theme itself was not necessarily dependent on quantifiable measures but in terms of whether it was coherent in capturing salient aspects in relation to the research aims. Similar themes from each transcript were identified and grouped before constructing overarching categories through an in-depth understanding of the relationship across existing themes. The research team would then raise and discuss any disagreements regarding the themes until a consensus was reached. Final themes were agreed among all the authors. Data analysis ended after achieving thematic saturation, whereby no new themes surfaced.

\section{Results}

A total of 30 interviews were conducted. We interviewed participants from a total of eight out of ten PCNs, and all participants recruited at that point of time were involved in the delivery of chronic care at the primary care setting.

\section{Participant characteristics}

Out of 30 participants recruited for our study, nine where PCN leaders. The average age of our participants was 49 years of age (range 31-68 years old), and their average duration spent in primary care was 18 years (range $3-35$ years). In fact, most participants had been in their own practice for a relatively long duration averaging 14 years (range 1-35 years). Our sample comprised of 27 male and three female private GPs.

\section{Main findings}

Three themes were identified as facilitators and two themes that were identified as barriers to the management of chronic conditions. The facilitators are 1) ancillary services to provide a "one-stop-shop", 2) chronic disease registry (CDR) to monitor care indicators and 3 ) funding for network. The barriers are 1) administrative burden of maintaining CDR and 2) loss of patients due to financial gradient in favour of public healthcare institutions.

\section{(+) Theme 1: Ancillary Services To Provide A "one-stop- shop"}


Every PCN is outfitted with wrap-around ancillary services which included diabetic retinal photography (DRP), diabetic foot screening (DFS) and nurse counselling (NC). These ancillary services enabled private practices which were traditionally too small to sustain or arrange for such services an opportunity to provide more holistic and preventive care for their patients. The individual practices are also supported by a team of nurses and care coordinators, expanding the time for patient care by the entire primary care team.

\section{Subtheme 1.1. Convenience of having the ancillary services arranged for patients}

Traditionally, private GPs would have to refer their patients to the polyclinics or government hospitals for ancillary services. However, the PCN enables each clinic to provide ancillary services to their patients when the services of a roving team are employed. Therefore, patients will not only be able to see the same doctor but also have the ancillary services conducted at the same location. As most patients reside near the clinic, this "one-stop-shop" enables a higher level of convenience and lowers the perceived barriers to attend ancillary services.

"[...] eye screening and nurse education [services available at polyclinics], but whereas in primary GP clinics, we are unable to offer that, right? So the current workflow is we need to refer patients back to even polyclinic or back to other service centres for eye screenings right? So these extra referrals or extra effort for the patient is not an optimal workflow for the patient and that will reduce the uptake on a lot of services. So PCN with our own ancillary and even mobile services, hopefully, it will provide a bit more convenience to the patient." (R26)

\section{Subtheme 1.2. Additional manpower provided for conducting and coordinating ancillary services}

The lack of ancillary service support and busy clinic hours raise the challenge of counselling patients on disease-modifying behaviours, diabetic eye and foot examinations for independent GPs. Having additional manpower in the form of nurses hired by the PCN contributes towards the practices in carrying out these essential ancillary services to prevent avoidable amputations or blindness in patients. PCNs have the liberty to either employ and train their own nurses or outsource the services to external vendors that provide a roving team of nurses to conduct ancillary services at their clinics.

Furthermore, PCNs are provided with additional manpower to arrange for ancillary services and remind patients to attend the arranged services. Having additional manpower to arrange for ancillary services and remind patients to attend the arranged services not only reduces the workload for clinic assistants but patients' default numbers. This task is fulfilled by primary care coordinators (PCCs), but it should be noted that PCCs can also be assigned other PCN related tasks such as the consolidation of patient data for maintenance of the CDR (elaborated in subtheme 3.2). 
"[...] CAs [clinic assistants] or my doctors will just have to register the patient, and then the PCCs will then follow up with patients on their appointments, and then they will book, and then they will then get their appointments, work with the patients to get their appointments and then bring the provider [roving ancillary services team] to provide their service in our clinic." (R46)

"The DRP, DFS I believe is done by Diabetic Society [external vendor]. Their nurses will be running inside the van. [Name of PCN] has their own roving nurse, so I understand that they will be providing the nurse counselling and also maybe helping with some of the DRP, DFS." (R21)

(+) Theme 2: Chronic disease registry to monitor care indicators

The CDR is a platform that enables the systematic tracking of care components for patients. Data is tabulated into an excel spreadsheet which comprises over 200 fields, from basic sociodemographic data, date of clinical diagnosis and screening attendances to clinical parameters for chronic conditions in accordance with local clinical practice guidelines. CDR data is submitted to AIC to ensure required care components are fulfilled before care plus fee (elaborated in subtheme 3.1) is dispersed by AIC to the PCN. Thus, the CDR gives GPs and AIC a dashboard view of the quality of care provided, allowing for the identification of opportunities to refine existing management practices using quantitative parameters by improving processes and outcomes.

\section{Subtheme 2.1. Cross accountability to ensure practices meet specific standards of care}

Most solo GP practices work in silos, and a certain level of accountability is needed to ensure that practices not only follow guidelines but provide the best care for their patients. Therefore, anonymized results of performance indicators of all practices are made available during implementation and review sessions every quarterly to perform benchmarking. This can potentially reduce the variation in performance and improve quality of care for patients.

"[...] my understanding of the CDR, is that they want to benchmark, they want to benchmark the care of the patient, that means ah, for example, the PCN, within the PCN, let's say, everybody, the average PCN for example, the HbA1c for diabetic, you know is let's say 8, and my clinic, all my patient are 9, then they say, I am below average, so there is a benchmarking [...]" (R39)

\section{Subtheme 2.2. Reminder to fulfil care processes}

This platform allows practices to follow-up with patients when required and ensure judicious completion of necessary procedures in their care management plans.

"Alright, so I guess in a way it [CDR] reminds especially the private doctors, especially when your clinic so busy. A lot of times we will overlook, or we will forget certain things [...] So this, in a way, is a constant reminder to making sure that this is done for the patient." (R26) 


\section{(+) Theme 3: Funding For The Network}

Every PCN is entitled to funding from the government. A commitment of $\$ 45$ million per annum by the government [30] will equip the network with more resources to better manage chronic patients than what could be harnessed as an individual private practice. This funding is mainly dispersed on a reimbursement basis through AIC.

\section{Subtheme 3.1. Care Plus Fee for extended consultation time}

Private practices are business entities that generate revenue mainly through consultation fee and the sale of medicines. As a result, the revenue generated is volume-based, making it more profitable for GPs to see to more acute cases. However, complex chronic patients require a lengthened consultation. Hence, the care plus fee (CPF) was introduced to reimburse clinics for extended consultation time. However, process and clinical outcome indicators stipulated in the CDR must be completed before the CPF is dispersed by AIC.

"The care plus fees are incentives for all members of the PCN [...] who are managing the complex chronic cases. They will receive a financial incentive per patient because it takes longer and more time resource to manage these patients. So per year, they are given a quantum of 100 dollars [per chronic patient], but of course, you must satisfy all the criteria [CDR requirements] stipulated to prove that you are managing a complex patient." (R15)

\section{Subtheme 3.2. Funding for additional manpower for backend office duties}

Funding is provided for PCNs to employ PCCs to coordinate ancillary services, track patients, remind patients to attend ancillary services and to consolidate data for the CDR as most solo practices do not have the manpower to conduct non-clinical duties. Funding for PCCs come in the form of full-time equivalents which is furnished by AIC based on the PCN's projected chronic patient load.

"Primary care coordinators if I am not mistaken [...] I think it is one is to one FTE [full-time equivalent] to 3000 patients [...]" (R36)

\section{Subtheme 3.3. Funding for locums for GPs to attend continuous medical education sessions}

Funding is provided for PCNs so that GPs can hire locums when they attend continuous medical education (CME) sessions. The availability of locums motivated GPs to attend CME sessions while maintaining clinic services in the interim.

"[...] I know that specifically there is funding for them to employ locum, so if they have to employ locum to go for this (CME sessions), there is funding to pay for their locum." (R48) 


\section{Subtheme 3.4. Funding for leads to perform PCN related duties}

Funding for PCN leads is used to backfill time lost at their practice when performing PCN related duties. Duties include developing working relationships with leads of other PCNs, providing strategic and clinical leadership and spearheading quality improvement over member practices.

"[...] PCN leads are given 0.4 FTE [full-time equivalent] [...] for a GP because it is an opportunity cost for you to be taken away from his clinic, that money goes directly into his pocket, that is to compensate him for the time lost because he could be otherwise seeing patients." (R36)

\section{(-) Theme 4: Administrative Burden Of Maintaining The CDR}

The maintenance of the CDR requires consolidation of data regarding the process and clinical indicators by both GPs and clinic assistants. Despite having additional administrative support from PCCs to consolidate registry data, routine documentation has proven highly laborious for practices overstretched by other administrative duties and lean manpower structure, leading to more man-hours or overtime duties. Clinics also face difficulty in extracting data from their clinical management system (CMS) due to the lack of a smart extraction tool that aligns with CDR requirements.

" Fortunately, our staff are understanding, but you cannot say so for the same for other solo practice, so the technical staff may not actually want to do paperwork, and if it falls on the onus of the doctors to do it, I don't think they have the time also beyond their clinical time too." (R18)

"For those clinics using Clinic Assist [CMS] with the CMS that is linked to PCN yes, that will be easier. You just need to key in your numbers and click submit [smart extraction tool function], but for a lot of other clinics not using Clinic Assist and integrated with PCN, what do we do? We need to manually write it down or manually key in individual patient clinical indicators for both MOH and PCN. So with the busy clinic, the doctor has no time to do it, the staff has no time to do it, so we need to OT [overtime] to submit all these." (R26)

(-) Theme 5: Loss of patients due to financial gradient in favour of public healthcare institutions

Perceptions of the affordability of healthcare affect the uptake of medical services. The high levels of government subsidies offered at public healthcare institutions such as the polyclinics and public specialist outpatient clinics are highly attractive to price-sensitive patients. The launch of Community Health Assist Scheme (CHAS), which extends healthcare cost subsidies to patients visiting private GPs attempts to alleviate the stress placed on the public healthcare sector resulting from the huge volume of patients drawn to their subsidized services and medicines.

\section{Subtheme 5.1. Insufficient CHAS quantum}


Complex chronic conditions require multiple visits to the clinic and long-term medication. Participants reflected that the quantum is usually sufficient for patients with simple chronic conditions but insufficient for patients with complex chronic conditions, as more medications need to be prescribed. Therein lies the possibility that care for multimorbid patients provided by their private GP might discontinue after the CHAS quantum has been exhausted.

"The CHAS subsidies help, but it is for simple chronic illness, for simple cases [...] But when it comes to more medication [...] it makes it very difficult, even with the CHAS subsidy." (R48)

\section{Subtheme 5.2. Heavily subsidized government-funded polyclinics}

The financial gradient between private primary care and government subvented primary care at polyclinics promotes specific health-seeking behaviour. As patients are price-sensitive, they turn to the largely government-funded polyclinics to obtain subsidized medications. Aside from cheaper medications, the heavily subsidized consultation fee makes polyclinics more appealing to priceconscious patients, promoting the severance in care continuity with their private GP.

"You cannot fight with the polyclinic because they are subsidized so you cannot compare. We have no subsidy for drugs. We have no subsidy for consultation." (R48)

"I would say that we have been able to keep a certain number of chronic patients within the registry. But of course, the challenge is keeping them in [...] they didn't come back after one visit, since last year. So this group of patients I would assume that they have kind of withdraw themselves from the system [...] Sometimes, they are, for example, going back to the polyclinic. Most of the time is cost issues." (R20)

\section{Discussion}

The provision of human and financial resources to upkeep the day to day operations of the PCN which includes the wrap-around ancillary services increase the accessibility of team-based care to patients and the use of the CDR to optimize care components are central in driving the PCN scheme forward. Ironically, the CDR, which is an enabler, also poses an administrative challenge for practices. Legacy issues regarding the financial gradient between private GP practices and polyclinics is another complex policy dilemma that requires further examination. Hereinafter the facilitators and barriers will be discussed in detail.

Ancillary services not traditionally offered by solo practices are now available through the PCN. The roving services provide DRP, DFS and NC, which are proven to be catalysts for preventing avoidable amputations and blindness [42-44]. The inconvenience caused to patients in making extra trips to polyclinics where ancillary services are offered, which resulted in missed attendances, was alleviated through roving teams that conduct the services at clinics [45]. A study by Schäfer et al. (2017) indicated that one-stop-services provided at GP clinics improved accessibility, continuity and comprehensiveness of 
care [46]. As GPs are usually burdened by assuming organizational and administrative tasks while providing medical care, assigning the responsibility of arranging and conducting ancillary services to someone else, such as the hiring of PCCs, allowed GPs to focus on the medical care for their patients [47-49]. This team-based care approach as studied through a meta-analysis by Levngood et al. (2019) established that team-based diabetes management improved overall clinical indicators for diabetes patients, health services utilization, diabetes-related morbidity and mortality [50].

Our participants supported the concept of the CDR, which allows for a certain level of benchmarking with other practices within the same PCN and track the process and clinical outcome indicators for their patients. A study by Luo et al. (2018) evaluated the effectiveness of diabetes management in a pilot PCN in Singapore [35]. The quantitative study briefly mentioned the use of a CDR but did not go into details as to how the CDR enabled better diabetes management. Our findings support the study by Luo et al. (2018) by elucidating how the CDR led practices to conform to higher levels of care standards. As defined by Schmittdiel et al. (2015), disease registries can serve to generate performance feedback reports on clinical outcomes; identify patients out of therapeutic range; create point-of-care reminders and decision support; and create "high-risk lists" that target patients who require more intensive management [51]. Other studies on electronic healthcare registries have suggested that disease documenting platforms if utilized in one or more of these ways as suggested by Schmittdiel et al. (2015), can improve care delivery for patients with diabetes [52-54].

Despite the advantages brought about by CDR, some barriers hinder its implementation. As reflected by our participants, the management of CDR is administratively burdensome, particularly for practices not supported by a CMS. Even for practices with a CMS, there is no smart extraction tool devoted to the exporting of CDR mandated fields [55]. As a result, the GPs or clinic assistants would have to key in the required CDR fields manually, resulting in additional man-hours or "overtime", as acknowledged by our participants. In addition, the need for increased documentation of care and coordination planning for patients as highlighted in a study by Gottschalk and Flocke (2005) might reduce the face-to-face time GPs have with patients [56].

A study by Stumm et al. (2019) illustrated that the lack of adequate compensation for the coordination of tasks hinders GPs from giving optimal care to their patients [47]. Therefore, funding for manpower to complete back-office tasks such as the coordination of ancillary services and consolidation of data fields for the CDR is allocated. Extra manpower such as having PCCs perform data retrieval and entry for the CDR would also translate to more face-to-face time for GPs with patients, resulting in better patient understanding and thus treatment of the condition. However, to our knowledge, there are other challenges to this, such as providing a space in the clinic for PCCs to work and the unwillingness of practices to grant access to patient data due to confidentiality issues.

Another facilitator that motivates GPs to manage more chronic patients is the CPF. Chronic patients typically require a longer consultation and more face-to-face time than primary care physicians customarily expend in routine practice $[48,57]$. If not compensated appropriately, chronic patients might 
not receive adequate consultation time, resulting in the omission of important standard-of-care items, reduced attention to patients' psychosocial concerns, and limited discussion of management options [58]. As private practices are profit-oriented entities, the compensation for additional time spent on a chronic patient is appropriate to offset the potential reduction in acute cases seen. However, our participants revealed the highly contingent nature of this funding model where CPF was dispersed only after the fulfilment of CDR requirements by religiously completing the necessary process and clinical outcome indicators. To our knowledge, CPF had only been distributed once since PCN's inception due to unspecified delays from AIC. We could only surmise that auditing of the fulfilment of CDR criteria took many man-hours at AIC's end as well. Nonetheless, the CPF is seen as augmentation for both the GP in terms of reimbursement for their time and the chronic patient who is ensured of evidence-based chronic disease care.

Given that PCNs operate around team-based care and a group of GPs, it is imperative for a strong leader to helm the network. Clinician leadership has been shown to be important in driving policy direction, strategic planning by operating across organization boundaries, and improving the practices within the network [59-61]. However, GPs might feel a strain taking on dual capacities, both as network leader and provider in their own practice. A study by Sephar et al. (2017) emphasized the challenges that GPs face between the clinical and leadership roles and a lack of formal training and preparation to assume the role of leader [62]. Thus, the reimbursement for their time in conducting duties as a leader for the network was paid accordingly, and the lack of leadership, management and financing skills of the GPs can be nurtured through a national health leadership model embedded into CME [63-64].

CME is essential for GPs to keep abreast of the latest chronic disease management practices and serve as a platform to exchange experiences with their colleagues [65]. In addition, PCN leaders are no longer only the captain of their practice but gatekeeper of the entire network. Thus, continuing professional development in clinical, business and financial leadership should be cornerstones for the development of PCN leaders [66]. In both instances, GPs are provided with funding to hire locums to fill their duties when attending CME courses. This motivates GPs to improve pre-existing levels of competence while having the manpower to cover their duties when they are absent from their practice.

Perceptions of the affordability of medical care undoubtedly affect the uptake of chronic care treatment [67]. In Singapore, CHAS was introduced as a portable medical subsidy to improve access to healthcare. It initially was only available for lower and middle-income Singaporeans, but from November 2019, all Singaporeans now enjoy CHAS benefits based on a tiered system whereby benefits are adjusted according to household monthly income and valuation of the home [68]. Recipients of this assistance scheme can seek subsidies for treatment from private GPs. All PCN clinics are CHAS enabled. More tiered subsidy schemes may motivate low SES patients to adhere to management plans and seek appropriate care [69]. Despite the enhancements made to CHAS to encourage Singaporeans to shift their care from polyclinics to the private GPs, our participants reflected that the CHAS quantum is insufficient to drive that behaviour. This is especially true for patients requiring multiple medications due to their complex conditions. All our participants had voiced that the high cost of unsubsidized medicines at private GPs 
had pushed patients to seek care at the heavily subsidized polyclinics. This financial gradient between private and public primary care institutions had long been the reason for patients sticking to polyclinics, especially in a healthcare system where services are mainly paid out-of-pocket and patients are free to choose their primary care provider [70].

Currently, there are 20 polyclinics in Singapore, with the number set to increase to 30 by $2030[71,72]$. Despite the introduction of CHAS, polyclinics continue to be confronted with high patient volumes [33, 73]. A study by Chow et al. (2012) demonstrated that affordability, the convenience of travel and onsite laboratory facilities influenced patients' choice of seeking treatment at polyclinics [70]. Enhancements to the convenience of onsite ancillary services at GP clinics will encourage more patients to seek services from their regular private GPs [70]. Thus, the inconvenience resulting from the lack of co-located ancillary services was resolved through the provision of roving ancillary services teams by the PCN. However, the

financial gradient with polyclinics remain. With the emergence of more polyclinics, privates GPs will find it increasingly difficult to compete for chronic patients who are price sensitive. This prevailing policy dilemma warrants further study. For now, we can only postulate that the increasing chronic burden might be too much for the consortia of private GPs alone to absorb, creating the need for more polyclinics.

\section{Strengths and limitations}

To our knowledge, this is the first time a qualitative study was conducted on the newly implemented PCN to explore the characteristics that make it a good model for chronic care management, in light of a growing ageing population with increased utilization of primary care services. Another strength is the participation of several authors in a rigorous process of data analysis and discussion until consensus on the themes was reached. We also managed to recruit participants from eight out of ten PCNs. Therefore, we believe that our findings are transferrable to all PCNs in Singapore as perspectives across PCNs should be similar, given the same contractual backbone for implementation and funding. However, we recognize that there could be a self-selection bias due to the nature of our recruitment strategy, whereby participants who had more positive experiences with the PCN might be inclined to participate in our study. Despite the potential one-sidedness in experiences, a range of views was demonstrated.

Our study illuminated the enablers and challenges of PCN from the providers' perspective. Moving forward, the next step is to evaluate the cost-effectiveness of PCN in managing chronic conditions compared to polyclinics and conduct studies on the facilitators and barriers of PCN from the patients' perspective.

\section{Conclusion}

The PCN initiative wields immense potential for the management of chronic diseases. The funding for streamlining back-office functions and increased manpower capacities to deliver a range of ancillary services to patients is a huge enabler for solo practices, who are now able to tap on more resources. Moreover, the CDR which tracks the patients' care delivery permits evidence-based care management. The 
challenges which include the administrative burden of maintaining the CDR need to be prioritized, and the financial gradient between private and public primary care systems partially surmounted through enhancements to CHAS remain to be addressed.

\section{Abbreviations}

AIC: Agency of Integrated Care; CDR: Chronic Disease Registry; CHAS: Community Health Assistance Scheme; CME: Continuous Medical Education; CMS: Clinic Management System; COREQ: COnsolidated criteria for Reporting Qualitative research; DFS: Diabetic Foot Screening; DRP: diabetic retinal photography; GP: General Practitioner; PCC: Primary Care Coordinator; PCN: Primary Care Network; MOH: Ministry of Health; MPH: Master of Public Health; NC: Nurse Counselling

\section{Declarations}

\section{Availability of data and materials}

Transcripts will not be shared to protect the anonymity of the GPs. Readers who wish to gain access to the data can write to the corresponding author; data may be granted upon reasonable request.

\section{Ethics approval and consent to participate}

Ethics approval was obtained from the National University of Singapore, Institutional Review Board (NUSIRB) before starting the study. The NUS-IRB reference code is S-19-005. A full explanation of the purpose and procedure of the study was provided to participants prior to obtaining their written informed consent. All demographic data and quotes used in this study were de-identified to maintain the anonymity of participants.

\section{Consent for publication}

Not applicable.

\section{Competing interests}

The authors declare that they have no competing interests.

\section{Funding}

This study was funded by two grants. The National Medical Research Council (NMRC) and Ministry of Health (MOH), Singapore, Health Service Research Grant (HSRG) (Funding Number:

NMRC/HSRG/0086/2018) and the Centre Grant Programme Singapore Population Health Improvement Center (Funding Number: NMRC/CG/C026/2017_NUHS). The funding organizations had no role in the 
study design, data collection and analysis, interpretation of the data, writing the paper and the decision to submit the paper for publication.

\section{Authors' contributions}

GK, DM, JC, TCH, FCD, SS and EH contributed to the conception and design of the research; FCD, SS and EH carried out the literature search; FCD and SS collected and analyzed the data; FCD drafted the manuscript and other authors revised it; DM acquired the grant to carry out this study. All authors read and approved the final manuscript. All authors agree to be accountable for all aspects of the work.

\section{Acknowledgements}

We would like to extend our gratitude to all participating GPs for sharing their thoughts and experiences on the PCN with us. We would also like to recognize the administrative support given by Rita Sim and Aloysius Chia of Duke-NUS Medical School and guidance from all other colleagues in the Enhancing Primary Care Team.

\section{References}

1. Quinn MP, Cardwell CR, Rainey A, McNamee PT, Kee F, Maxwell AP, et al. The Impact of Admissions for the Management of End-Stage Renal Disease on Hospital Bed Occupancy. Nephron Clin Pract. 2009;113(4):c315-20.

2. Mihailoff M, Deb S, Lee JA, Lynn J. The Effects of Multiple Chronic Conditions on Adult Patient Readmissions and Hospital Finances: A Management Case Study. Inq J Med Care Organ Provis Financ. 2017;01:54:46958017729597.

3. Mannino DM, Homa DM, Akinbami LJ, Ford ES, Redd SC. Chronic obstructive pulmonary disease surveillance-United States, 1971-2000. Morb Mortal Wkly Rep Surveill Summ Wash DC 2002. 2002 Aug 2;51(6):1-16.

4. Sampson MJ, Dozio N, Ferguson B, Dhatariya K. Total and excess bed occupancy by age, specialty and insulin use for nearly one million diabetes patients discharged from all English Acute Hospitals. Diabetes Res Clin Pract. 2007 Jul;77(1)(1):92-8.

5. Other Chronic Diseases Threaten Health and Economies in Poor and Middle-Income Countries

\section{World Bank Report}

Obesity D, Attacks H. and Other Chronic Diseases Threaten Health and Economies in Poor and Middle-Income Countries - World Bank Report. 2011. https://www.worldbank.org/en/news/pressrelease/2011/09/15/obesity-diabetes-heart-attacks-other-chronic-diseases-threaten-healtheconomies-poor-middle-income-countries-world-bank-report. Accessed 9 April 2020. 
6. Donnan PT, Leese GP, Morris AD. Diabetes Audit and Research in Tayside, Scotland/Medicine Monitoring Unit Collaboration. Hospitalizations for people with type 1 and type 2 diabetes compared with the nondiabetic population of Tayside, Scotland: a retrospective cohort study of resource use. Diabetes Care. 2000 Dec;23(12):1774-9.

7. Wolff JL, Starfield B, Anderson G. Prevalence, expenditures, and complications of multiple chronic conditions in the elderly. Arch Intern Med. 2002 Nov;11(20):2269-76. 162(.

8. Freund T, Campbell SM, Geissler S, Kunz CU, Mahler C, Peters-Klimm F, et al. Strategies for Reducing Potentially Avoidable Hospitalizations for Ambulatory Care-Sensitive Conditions. Ann Fam Med. 2013 Jan;7(4):363-70. 11(.

9. Reynolds R, Dennis S, Hasan I, Slewa J, Chen W, Tian D, et al. A systematic review of chronic disease management interventions in primary care. BMC Fam Pract. 2018 Jan 9;19(1):11.

10. McPhail SM. Multimorbidity in chronic disease: impact on health care resources and costs. Risk Manag Healthc Policy Macclesfield. 2016;9:143-56.

11. Bodenheimer T, Wagner EH, Grumbach K. Improving primary care for patients with chronic illness. JAMA. 2002 Oct 9;288(14):1775-9.

12. Dusheiko M, Gravelle H, Martin S, Rice N, Smith PC. Does better disease management in primary care reduce hospital costs? Evidence from English primary care. J Health Econ. 2011 Sep;30(5)(1):91932.

13. Rothman AA, Wagner EH. Chronic illness management: what is the role of primary care? Ann Intern Med. 2003 Feb;4(3):256-61. 138(.

14. Katon WJ, Lin EHB, Von Korff M, Ciechanowski P, Ludman EJ, Young B, et al. Collaborative care for patients with depression and chronic illnesses. N Engl J Med. 2010 Dec;30(27):2611-20. 363(.

15. Boult C, Wieland GD. Comprehensive Primary Care for Older Patients With Multiple Chronic Conditions: “Nobody Rushes You Through.” JAMA. 2010 Nov 3;304(17):1936-43.

16. Wagner $\mathrm{EH}$. The role of patient care teams in chronic disease management. BMJ. 2000 Feb;26(7234):569-72. 320(.

17. Smith CD, Balatbat C, Corbridge S, Dopp AL, Fried J, Harter R, et al. Implementing Optimal TeamBased Care to Reduce Clinician Burnout. NAM Perspect. 2018. https://nam.edu/implementingoptimal-team-based-care-to-reduce-clinician-burnout/. Accessed 9 April 2020.

18. Alberta PCNs - Primary Care Networks Program Management Office. 2015. https://pcnpmo.ca/alberta-pcns/Pages/default.aspx. Accessed 9 April 2020.

19. Primary Care Network (PCN). 2020. https://www.primarycarepages.sg/practicemanagement/primary-care-model/primary-care-network-(pcn). Accessed 28 March 2020.

20. Hutchison B, Levesque J-F, Strumpf E, Coyle N. Primary health care in Canada: systems in motion. Milbank Q. 2011 Jun;89(2):256-88.

21. Cumming J. Integrated care in New Zealand (Special 10th Anniversary Edition). Int J Integr Care. 2011 Nov 18;11. 
22. Ose D, Kamradt M, Kiel M, Freund T, Besier W, Mayer M, et al. Care management intervention to strengthen self-care of multimorbid patients with type 2 diabetes in a German primary care network: A randomized controlled trial. PloS One. 2019;14(6):e0214056.

23. Schlette S, Lisac M, Blum K. Integrated primary care in Germany: the road ahead. Int J Integr Care. 2009 Apr 20;9.

24. McAlister FA, Bakal JA, Green L, Bahler B, Lewanczuk R. The effect of provider affiliation with a primary care network on emergency department visits and hospital admissions. CMAJ. 2018 Mar 12;190(10):E276-84.

25. Manns BJ, Tonelli M, Zhang J, Campbell DJT, Sargious P, Ayyalasomayajula B, et al. Enrolment in primary care networks: impact on outcomes and processes of care for patients with diabetes. CMAJ Can Med Assoc J. 2012 Feb 7;184(2):E144-52.

26. Johnson JA, Sayah FA, Wozniak L, Rees S, Soprovich A, Chik CL, et al. Controlled trial of a collaborative primary care team model for patients with diabetes and depression: Rationale and design for a comprehensive evaluation. BMC Health Serv Res. 2012 Dec;12(1):1-12.

27. Johnson JA, Lier DA, Soprovich A, Al Sayah F, Qiu W, Majumdar SR. Cost-Effectiveness Evaluation of Collaborative Care for Diabetes and Depression in Primary Care. Am J Prev Med. 2016 Jul;1(1):e1320. 51 .

28. Mills J, Oyedotun L, Ridout J, Holmes M. The opportunities for economies of scale in primary care. InnovAiT. 2019 Aug 1;12(8):476-8.

29. $\mathrm{MOH}$ | News Highlights. https://www.moh.gov.sg/news-highlights/details/primary-care-networks-forbetter-patient-care-in-the-community. Accessed 23 Feb 2020.

30. Khoo HS, Lim YW, Vrijhoef HJ. Primary healthcare system and practice characteristics in Singapore. Asia Pac Fam Med. 2014 Dec;13(1):1-8.

31. Cheah J, Kirk-Chuan W, Lim H. Integrated care: from policy to implementation - The Singapore Story. Int J Integr Care. 2012 Sep 4;12.

32. $\mathrm{MOH}$ | Primary Healthcare Services [Internet]. https://www.moh.gov.sg/home/our-healthcaresystem/healthcare-services-and-facilities/primary-healthcare-services. Accessed 20 March 2020.

33. Tan KB, Lee CE. Integration of Primary Care with Hospital Services for Sustainable Universal Health Coverage in Singapore. Health Syst Reform. 2019 Jan 2;5(1):18-23.

34. $\mathrm{MOH}$ | Primary Care Survey. 2014 Report. https://www.moh.gov.sg/resourcesstatistics/reports/primary-care-survey-2014-report. Accessed 28 March 2020.

35. Luo M, Poh Z, Koh G, Tham TY, Lau W, Toh SA, et al. Diabetes management in a Primary Care Network (PCN) of private general practitioners in Singapore: An observational study. Medicine (Baltimore). 2018 Oct;97(43):e12929.

36. Chua LKL, Chong CK, Hwee-Lin W, Yean TT. Primary Care Network (PCN) As A Model Of Care For GP Chronic Disease Management. Singap Fam Physician. 2015 Jul;41(2)(1):61-4. 
37. Tracy SJ. Qualitative Quality: Eight “Big-Tent” Criteria for Excellent Qualitative Research. Qual Inq. 2010 Dec;16(10):837-51.

38. Pope $C$, Mays N. Reaching the parts other methods cannot reach: an introduction to qualitative methods in health and health services research. BMJ. 1995 Jul;1(6996):42-5. 311 (.

39. Tong A, Sainsbury P, Craig J. Consolidated criteria for reporting qualitative research (COREQ): a 32item checklist for interviews and focus groups. Int J Qual Health Care J Int Soc Qual Health Care. 2007 Dec;19(6):349-57.

40. Davidsen AS. Phenomenological Approaches in Psychology and Health Sciences. Qual Res Psychol. 2013 Jul 1;10(3):318-39.

41. Braun V, Clarke V. Using thematic analysis in psychology. Qual Res Psychol. 2006 Jan 1;3(2):77-101.

42. Nather A, Cao S, Chen JLW, Low AY. Prevention of diabetic foot complications. Singapore Med J. 2018 Jun;59(6):291-4.

43. Mayfield JA, Reiber GE, Nelson RG, Greene T. Do foot examinations reduce the risk of diabetic amputation? J Fam Pract. 2000 Jun;49(6):499-504.

44. Skaggs JB, Zhang X, Olson DJ, Garg S, Davis RM. Screening for Diabetic Retinopathy: Strategies for Improving Patient Follow-up. N C Med J. 2017 Mar 1;78(2):121-3.

45. Taber JM, Leyva B, Persoskie A. Why do People Avoid Medical Care? A Qualitative Study Using National Data. J Gen Intern Med. 2015 Mar;30(3):290-7.

46. Schäfer WLA, Boerma WGW, Schellevis FG, Groenewegen PP. GP Practices as a One-Stop Shop: How Do Patients Perceive the Quality of Care? A Cross-Sectional Study in Thirty-Four Countries. Health Serv Res. 2018 Aug;53(4):2047-63.

47. Stumm J, Thierbach C, Peter L, Schnitzer S, Dini L, Heintze C, et al. Coordination of care for multimorbid patients from the perspective of general practitioners - a qualitative study. BMC Fam Pract. 2019 Nov;20(1):160. 20(.

48. Østbye T, Yarnall KSH, Krause KM, Pollak KI, Gradison M, Michener JL. Is There Time for Management of Patients With Chronic Diseases in Primary Care? Ann Fam Med. 2005 May;3(3):209-14.

49. Margolius D, Wong J, Goldman ML, Rouse-Iniguez J, Bodenheimer T. Delegating Responsibility from Clinicians to Nonprofessional Personnel: The Example of Hypertension Control. J Am Board Fam Med. 2012 Mar;25(2)(1):209-15.

50. Levengood TW, Peng Y, Xiong KZ, Song Z, Elder R, Ali MK, et al. Team-Based Care to Improve Diabetes Management: A Community Guide Meta-analysis. Am J Prev Med. 2019;57(1):e17-26.

51. Schmittdiel J, Bodenheimer T, Solomon NA, Gillies RR, Shortell SM. Brief report: The prevalence and use of chronic disease registries in physician organizations. A national survey. J Gen Intern Med. 2005 Sep;20(9):855-8.

52. Stroebel RJ, Scheitel SM, Fitz JS, Herman RA, Naessens JM, Scott CG, et al. A randomized trial of three diabetes registry implementation strategies in a community internal medicine practice. $\mathrm{Jt}$ 
Comm J Qual Improv. 2002 Aug;28(8):441-50.

53. Hoque DME, Kumari V, Hoque M, Ruseckaite R, Romero L, Evans SM. Impact of clinical registries on quality of patient care and clinical outcomes: A systematic review. PLoS ONE. 2017 Sep 8;12(9).

54. Thomas KG, Thomas MR, Stroebel RJ, McDonald FS, Hanson GJ, Naessens JM, et al. Use of a Registry-generated Audit, Feedback, and Patient Reminder Intervention in an Internal Medicine Resident Clinic-A Randomized Trial. J Gen Intern Med. 2007 Dec;22(12):1740-4.

55. IHis. SmartCMS Programme. 2020. https://www.ihis.com.sg/SmartCMS_Programme. Accessed 9 April 2020.

56. Gottschalk A, Flocke SA. Time Spent in Face-to-Face Patient Care and Work Outside the Examination Room. Ann Fam Med. 2005 Nov;3(6):488-93.

57. Schellevis FG, Van de Lisdonk EH, Van der Velden J, Hoogbergen SH, Van Eijk JT, Van Weel C. Consultation rates and incidence of intercurrent morbidity among patients with chronic disease in general practice. Br J Gen Pract. 1994 Jun;44(383):259-62.

58. Barnes CS, Ziemer DC, Miller CD, Doyle JP, Watkins C, Cook CB, et al. Little Time for Diabetes Management in the Primary Care Setting. Diabetes Educ. 2004 Jan;30(1)(1):126-35.

59. Willcocks SG. Leadership: a challenge for GPs? Br J Health Care Manag. 2010 Oct;16(10):468-73.

60. Storey J, Holti R, Hartley J, Marshall M. Devolving healthcare services redesign to local clinical leaders: does it work in practice? J Health Organ Manag Bradf. 2019;33(2):188-203.

61. Weaver RR. Seeking high reliability in primary care: Leadership, tools, and organization. Health Care Manage Rev. 2015;40(3):183-92.

62. Spehar I, Sjøvik H, Karevold KI, Rosvold EO, Frich JC. General practitioners' views on leadership roles and challenges in primary health care: a qualitative study. Scand J Prim Health Care. 2017 Mar 1;35(1):105-10.

63. Swanwick T, Varnam R. Leadership development and primary care. BMJ Lead. 2019 Jun 1;3(2). Available from: https://bmjleader.bmj.com/content/3/2/59. Accessed 9 April 2020.

64. Hargett CW, Doty JP, Hauck JN, Webb AM, Cook SH, Tsipis NE, et al. Developing a model for effective leadership in healthcare: a concept mapping approach. J Healthc Leadersh. 2017 Aug 28;9:69-78.

65. Kjaer NK, Steenstrup AP, Pedersen LB, Halling A. Continuous professional development for GPs: experience from Denmark. Postgrad Med J. 2014 Jul;1(1065):383-7. 90(.

66. Raza A, Coomarasamy A, Khan KS. Best evidence continuous medical education. Arch Gynecol Obstet. 2009 Oct;280(4)(1):683-7.

67. Vuong Q-H, Ho T-M, Nguyen H-K, Vuong T-T. Healthcare consumers' sensitivity to costs: a reflection on behavioural economics from an emerging market. Palgrave Commun. 2018 Jun 19;4(1):1-10.

68. More to benefit from revised income criteria for healthcare subsidy schemes. 2019. https://www.chas.sg/news_events_article.aspx?id=1535. Accessed 21 March 2020.

69. Chan CQH, Lee KH, Low LL. A systematic review of health status, health seeking behaviour and healthcare utilization of low socioeconomic status populations in urban Singapore. Int J Equity 
Health. 2018 Apr 2;17(1):39.

70. Chow WL, Wang VW, Low YS, Tse DWL, Lim JFY. Factors that influence the choice of seeking treatment at polyclinics. Singapore Med J. 2012 Feb;53(2):109-15.

71. Parliament: Six new polyclinics by 2023 , with up to six more by 2030 , Health News \& Top Stories The Straits Times. 2019. https://www.straitstimes.com/singapore/health/six-new-polyclinics-by2023-with-up-to-six-more-by-2030. Accessed 9 April 2020.

72. $\mathrm{MOH}$ to build up to 8 new polyclinics by 2030. CNA. 2019. https://www.channelnewsasia.com/news/singapore/moh-to-build-up-to-8-new-polyclinics-by-203010022706. Accessed 9 April 2020.

73. Lim M-K. Shifting the burden of health care finance: a case study of public-private partnership in Singapore. Health Policy. 2004 Jul;69(1)(1):83-92.

\section{Figures}




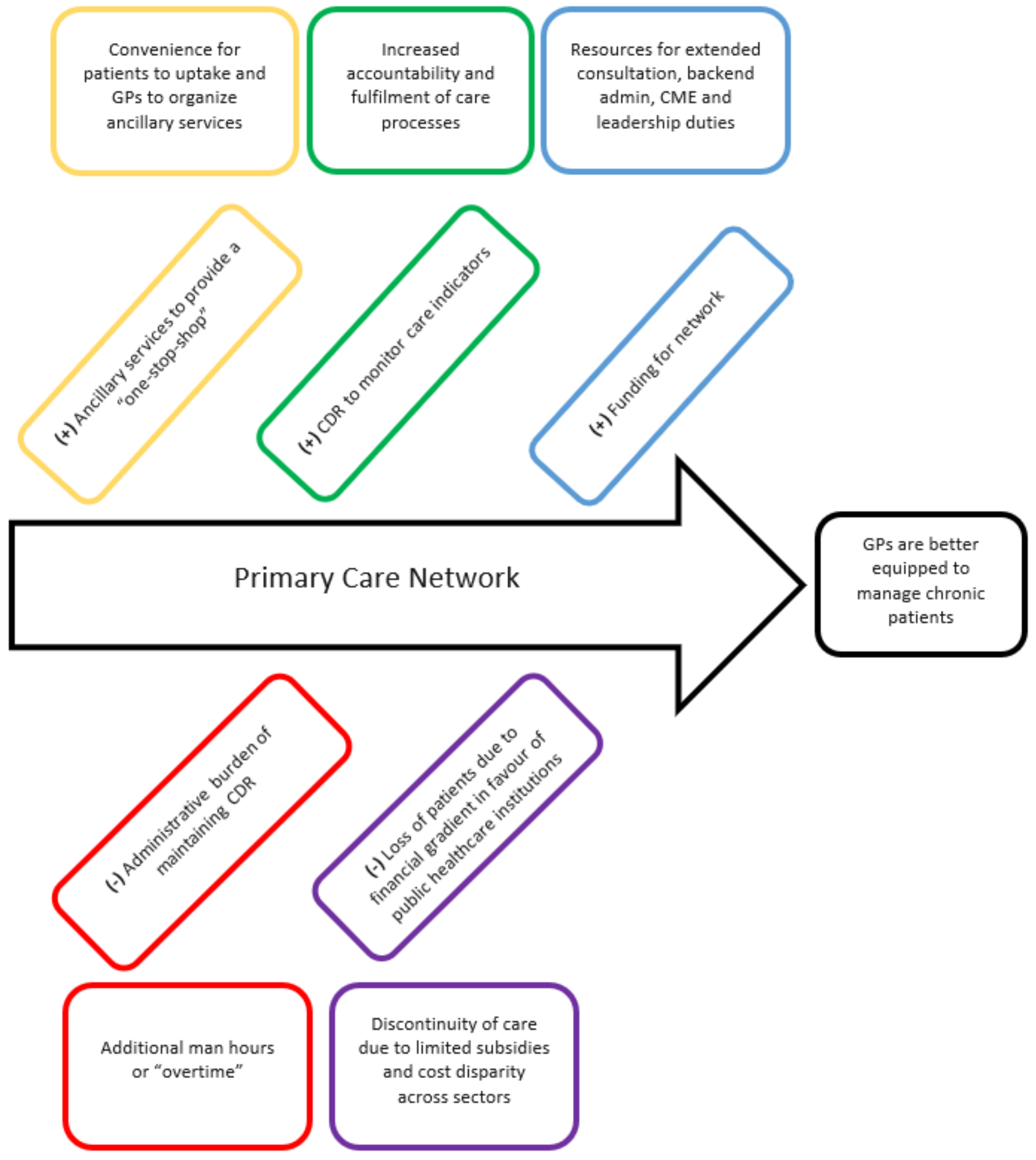

Figure 1

Diagram of facilitators (+) and barriers (-) developed based on results

\section{Supplementary Files}

This is a list of supplementary files associated with this preprint. Click to download. 
- Additionalfile1.docx

- Additionalfile2.docx

Page 23/23 